\title{
Preventive Gabapentin versus Pregabalin to Decrease Postoperative Pain after Lumbar Microdiscectomy: A Randomized Controlled Trial
}

\author{
Mohsin Qadeer ${ }^{1}$, Muhammad Waqas ${ }^{1}$, Muhammad Jawad Rashid ${ }^{2}$, \\ Syed Ather Enam ${ }^{1}$, Salman Sharif ${ }^{3}$, Ghulam Murtaza ${ }^{4}$ \\ ${ }^{1}$ The Aga Khan University Hospital, Karachi, Pakistan \\ ${ }^{2}$ Baqai Medical College, Karachi, Pakistan \\ ${ }^{3}$ Liaquat National Hospital, Karachi, Pakistan \\ ${ }^{4}$ Patel Hospital, Karachi, Pakistan
}

Study Design: Randomized controlled trial.

Purpose: The purpose of this study was to compare pregabalin and gabapentin for mean postoperative visual analog score (VAS) for pain in patients undergoing single-level lumbar microdiscectomy for intervertebral disc prolapse at a tertiary care hospital.

Overview of Literature: Pregabalin has a superior pharmacokinetic profile and analgesic effect at lower doses than gabapentin; however, analgesic efficacy must be established during the perioperative period after lumbar spine surgery.

Methods: This randomized controlled trial was carried out at our institute from February to October 2011 on 78 patients, with 39 participants in each study group. Patients undergoing lumbar microdiscectomy were randomized to group A (gabapentin) or group B (pregabalin) and started on trial medicines one week before surgery. The VAS for pain was recorded at 24 hours and one week postoperatively.

Results: Both groups had similar baseline variables, with mean ages of 42 and 39 years in groups $A$ and $B$, respectively, and a majority of male patients in each group. The mean VAS values for pain at 24 hours for gabapentin vs. pregabalin were comparable $(1.97 \pm 0.84$ vs. $1.6 \pm 0.87$, respectively; $p=0.087)$ as were the results at one week after surgery $(0.27 \pm 0.45$ vs. $0.3 \pm 0.46$, respectively; $p=0.79)$. None of the patients required additional analgesia postoperatively. After adjusting for age and sex, the VAS value for group B patients was 0.028 points lower than for group A patients, but this difference was not statistically significant $\left(p=0.817, R^{2}=0.018\right)$.

Conclusions: Pregabalin is equivalent to gabapentin for the relief of postoperative pain at a lower dose in patients undergoing lumbar microdiscectomy. Therefore, other factors, such as dose, frequency, cost, pharmacokinetics, and side effects of these medicines, should be taken into account whenever it is prescribed.

Keywords: Intervertebral disc displacement; Lumbar vertebrae; Pregabalin; Gabapentin; Postoperative pain

\section{Introduction}

Effective pain control after major surgical procedures not only affects patient satisfaction but also the morbidity and length of hospital stay [1-3]. Prevention and treatment of postoperative pain continue to be major challenges in

Received May 20, 2016; Revised Jul 10, 2016; Accepted Jul 22, 2016

Corresponding author: Ghulam Murtaza

Consultant in General Surgery, Patel Hospital, Gulshan-e-Iqbal, Karachi, Pakistan

Tel: +923043546377, E-mail: shaikh.murtaza@outlook.com 
postoperative care for humanitarian, ethical, and medical reasons [4]. One of the most important predictors of chronic postoperative pain is the severity of acute postoperative pain [5].

Despite major advancements in the understanding of pain and its control, almost $80 \%$ patients still experience significant postoperative pain [6]. The risk of inappropriate pain control is especially high in patients undergoing surgery for chronic low back pain, who often develop tolerance to strong analgesics [7]. The use of multimodal treatments has reduced the side effects and doses required for adequate analgesia [8-10]. Multiple mechanisms suggest that a combination of different analgesics will reduce side effects and opioid dependence and will synergistically enhance analgesia [8].

Pregabalin is a structural analog of gamma-aminobutyric acid (GABA) and shares some characteristics with its precursor gabapentin. Its mechanism of action is the same as gabapentin, but it has a superior pharmacokinetic profile [11]. Its efficacy for the treatment of acute pain is similar to that of gabapentin; however, it has greater bioavailability, linear pharmacokinetics, requires lower dose titration, and has a shorter time required to achieve an optimal analgesic effect $[12,13]$. Although structurally similar to gabapentin, pregabalin has greater analgesic efficacy in rodent models of neuropathic pain [14,15] with lower intersubject variability. Pregabalin also appears to be more effective for the control of acute nociceptive pain after surgery and the reduction of both opioid dependence and anxiety $[16,17]$. In previous studies, the visual analog score (VAS) values for pain 24 hours after surgery for gabapentin versus pregabalin were $3.6 \pm 1.4$ [18] versus $1.73 \pm 2.02$ [19] (mean \pm standard deviation).

A few studies have addressed the use of pregabalin as part of an analgesic regimen for lumbar spine surgery specifically. However, pregabalin was either compared to placebo or there were limitations in the study design, according to the pharmacological properties of the drugs, i.e., the drug was used just before the surgery, but it requires at least a week to yield effective analgesia [20]. Thus, interventional drugs should be administered one week prior to surgery for maximal benefit at the time of surgery.

Because the preventive use of GABA analogs before surgery reduces both side effects and the need for postoperative analgesia, both gabapentin and pregabalin should be compared in the same study to determine the drug with the more favorable outcome (better safety and pharma- cokinetic profiles) based on valid evidence. Therefore, we intended to compare lower doses of pregabalin to gabapentin in terms of the mean VAS value for postoperative pain in patients undergoing single-level lumbar microdiscectomy for intervertebral disc prolapse at a tertiary care hospital. We hypothesized that the pain in the pregabalin group would be less than that in the gabapentin group.

\section{Materials and Methods}

This parallel-group, single-blinded, randomized controlled trial was conducted by the department of neurosurgery, Aga Khan University Hospital, from February to October 2011, and included 78 patients who underwent lumbar microdiscectomy. The Ethical Review Committee (173-SUR-ERC-10) approved this study (Registered at Clinical Trials.gov [NCT02120703]). Patients between 30 and 60 years of age who underwent elective lumbar microdiscectomy for intervertebral disc prolapse were randomized to either group A (gabapentin, trade name: neogab, Hilton Pharma, Pakistan) or group B (pregabalin, trade name: zeegap, Hilton Pharma, Karachi, Pakistan) using a computer-generated table for the desired sample size. To reduce confounding effects, we excluded patients with the following: (1) microdiscectomy at two or more vertebral levels, (2) use of instrumentation (e.g., pedicle screw fixation, hooks, etc.), (3) history of steroids or alcohol use, (4) multiple comorbidities (e.g., renal failure, chronic liver disease, etc.), (5) use of anticonvulsant drugs, (6) spinal deformity, (7) obesity (body mass index >30), (8) inability to understand and respond to the VAS, and (9) known allergy to GABA analogs.

We calculated sample size using World Health Organization software that employed the formula for hypothesis testing of two population means (one-sided). In previous studies, the VAS values for pain 24 hours after surgery for gabapentin versus pregabalin were $3.6 \pm 1.4$ [18] versus $1.7 \pm 2.02$ [19] (mean \pm standard deviation). At a 5\% level of significance and a $99 \%$ power, a sample size of 36 was required for each intervention arm. With an expected dropout rate of $10 \%$ (three subjects per group), 39 patients were enrolled per group.

Informed consent was obtained in the preoperative clinic upon registration of the patient for surgery. A computer-generated randomization sequence was generated by a statistician. Patients were administered pregabalin or gabapentin one week before surgery at doses of $75 \mathrm{mg}$ 
and $200 \mathrm{mg}$ twice daily, respectively. Two weeks' worth of medication was supplied by the research team in a sealed opaque envelope with the randomization number written on it. Trial medicines were continued postoperatively, along with tramadol (50 mg, three times a day), $500 \mathrm{mg}$ paracetamol+pseudoephedrine (one tablet, three times a day) and ibuprofen (400 mg, three times a day). Patients were also offered morphine as patient-controlled intermittent analgesia (PCIA); any additional analgesia used in 24 hours in the form of PCIA was recorded (effect modifier).

Lumbar microdiscectomy was performed by a single surgeon. Postoperatively, all patients were mobilized after 24 hours with the help of a physiotherapist and ambulated at least 15-20 meters. Immediately thereafter, patients were approached by a registered nurse to register the VAS value for pain. The nurse was unaware of the trial medicine and hypothesis. Patients were discharged on the second postoperative day, prescribed the same medications mentioned above, and followed up in the clinic after one week. Patient was mobilized in the clinic and VAS was recorded by a registered nurse. Patients were discharged from the trial thereafter, and medications were continued at the discretion of the primary surgeon.

SPSS ver. 16 (SPSS Inc., Chicago, IL, USA) was used for data analysis. Continuous variables (i.e., age, pain score, and postoperative analgesia consumption) were reported as the mean \pm standard deviation. Categorical variables (i.e., sex) were reported as proportions. Outcomes (i.e., VAS values after 1 day and 1 week) were compared between the groups by the student's $t$-test. Confounding and effect modifying variables (i.e., age, sex, and amount of additional analgesia needed) were analyzed by multiple linear regression. $p$-values less than 0.05 were considered statistically significant.

\section{Results}

A total of 122 patients were evaluated, and 78 patients were enrolled in the study. There were 39 participants in each group (Fig. 1). Both groups were comparable for baseline demographic variables (Table 1).

The mean VAS values for pain for both groups were comparable, both 24 hours and one week after surgery (24 hours: $1.97 \pm 0.84$ vs. $1.6 \pm 0.87, p=0.087$ and 1 week: $0.27 \pm 0.45$ vs. $0.3 \pm 0.46, p=0.79$, respectively) (Table 2 ). No patient required additional analgesia postoperatively. Patients in group A reported increased thirst compared

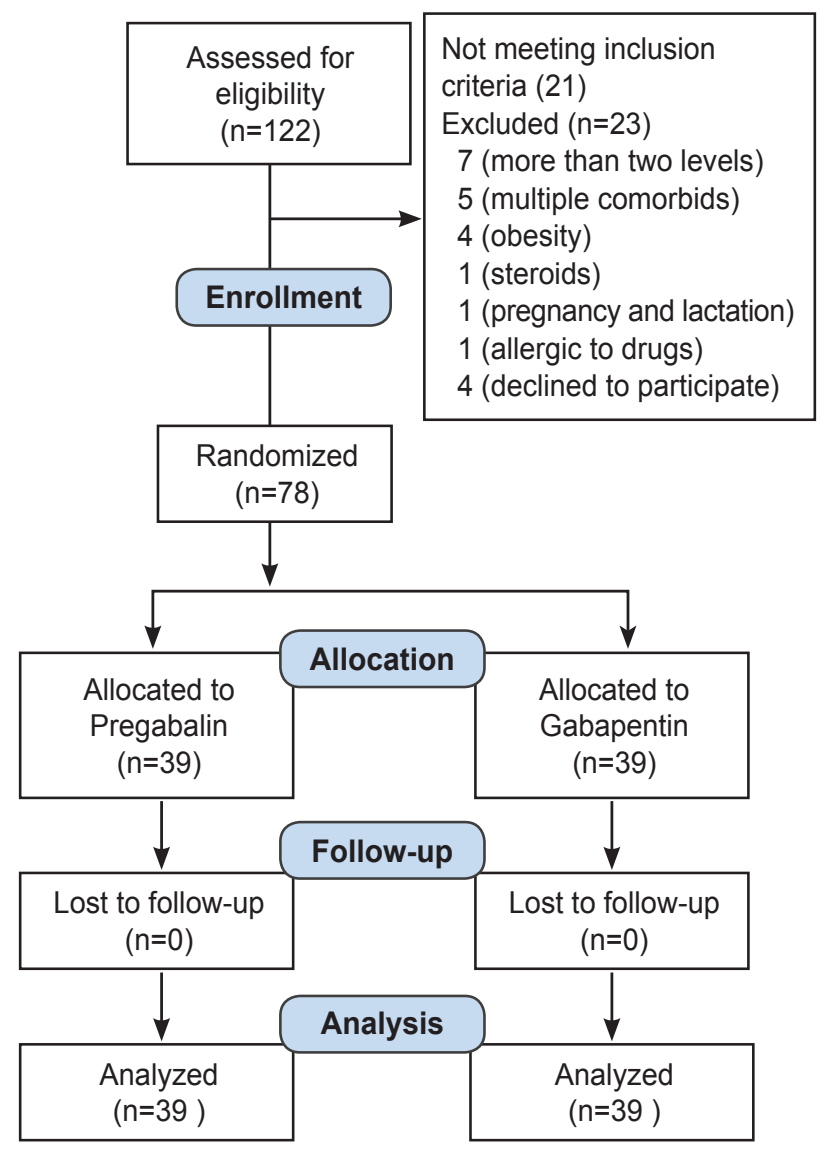

Fig. 1. Flow diagram of participants.

Table 1. Baseline comparison of variables

\begin{tabular}{lcc}
$\begin{array}{l}\text { Variable } \\
\text { Age }\end{array}$ & $\begin{array}{c}\text { Gabapentin } \\
(\mathrm{n}=39)\end{array}$ & $\begin{array}{r}\text { Pregabalin } \\
(\mathrm{n}=39)\end{array}$ \\
\hline Sex & $42 \pm 8.9$ & $39 \pm 12$ \\
\hline Female & $12(30)$ & $10(25)$ \\
\hline Male & $27(70)$ & $29(75)$ \\
\hline Body mass index & $26.8 \pm 2.9$ & $27.1 \pm 1.9$ \\
\hline Comorbids & & \\
\hline Diabetes mellitus & $4(10.2)$ & $5(12.8)$ \\
\hline Hypertension & $5(12.8)$ & $3(7.6)$ \\
\hline COPD & $1(2.5)$ & $1(2.5)$ \\
\hline Others & $3(7.6)$ & $4(10.2)$ \\
\hline Duration of symptoms (wk) & $6.7 \pm 2.9$ & $7.3 \pm 3.5$ \\
\hline
\end{tabular}

Values are presented as number (\%).

COPD, chronic obstructive pulmonary disease.

to those in group B; otherwise, no adverse effects were reported by the research participants.

The multivariable linear regression model of the VAS 
Table 2. Outcome variables

\begin{tabular}{lccc} 
Outcome & Gabapentin & Pregabalin & $p$-value \\
VAS at 24 hours & $1.97 \pm 0.84$ & $1.6 \pm 0.87$ & $0.087^{\text {al }}$ \\
VAS at 1 week & $0.27 \pm 0.45$ & $0.3 \pm 0.46$ & 0.79 \\
\hline
\end{tabular}

Values are presented as number (\%).

VAS, visual analog score.

${ }^{\text {a) }}$ Student's $t$-test.

for pain at 1 week consisted of the main exposure (intervention) and covariates (age and sex). After adjusting for age and sex, the VAS value for group B patients was 0.028 points lower than that for the group A patients, but this difference was not statistically significant ( $p=0.817$, $\left.R^{2}=0.018\right)$.

\section{Discussion}

This randomized controlled trial compared pregabalin and gabapentin for mean postoperative pain in patients undergoing single-level lumbar microdiscectomy for intervertebral disc prolapse. We found that both drugs were equivalent; thus, we failed to prove our hypothesis that the pain in the pregabalin group would be less than that in the gabapentin group.

The concept of multimodal pain management, that is, the reduction of adverse effects due to lower doses of pain medications, has evolved over time [21]. After spinal surgery, opioids and nonsteroidal anti-inflammatory drugs have been the standards of treatment. The use of these drugs at high doses, however, has been associated with side effects, like drowsiness, constipation, urinary retention, etc. $[10,21]$, which has led to the popularity and frequent use of newer, anticonvulsant therapies [22].

Pregabalin and its precursor, gabapentin, are analogs of the inhibitory neurotransmitter, GABA. These compounds have anticonvulsant, antihyperalgesic, and anxiolytic effects, and both bind to the alpha 2-delta ( $\alpha 2-\delta)$ subunit of presynaptic, voltage-gated calcium channels. The probable mechanism of action of pregabalin/gabapentin is to reduce the release of excitatory neurotransmitters (e.g., glutamate, substance $\mathrm{P}$, calcitonin, noradrenaline, generelated peptide) by inhibiting calcium influx via calcium channels [23].

The pharmacological properties of pregabalin, however, are not identical to those of gabapentin. Although structurally similar to gabapentin, pregabalin has greater analgesic efficacy. Pregabalin is pharmacologically superior to gabapentin due to its higher bioavailability (90\% vs. $33 \%-$ 66\%), more rapid absorption (peak plasma level: 1 hour vs. 3-4 hours), and linear increase in plasma concentrations when its dose is increased [11]. The lower doses of pregabalin required for its analgesic effect, compared to gabapentin, result in better tolerance and fewer side effects, making its use more advantageous. Although not a part of this study, the observation was made that, even at these low doses, patients on gabapentin complained of thirst. No major side effects were reported by patients in the pregabalin group at these doses; however, the sample size in our study is too small to make conclusions about the side effect profile.

Multiple studies have shown the beneficial effects of pregabalin and gabapentin administered just before surgery, compared to their postoperative administration [19]. The CSF levels of pregabalin required to elicit the desired amount of pain reduction may require from 6 hours to one week after oral administration [24] and the beneficial pain controlling effects may take up to 1 week. We thus designed our study to administer pregabalin to patients at least one week before surgery, so as to obtain its maximum analgesic effects. We also used a lower dose of pregabalin to decrease any possible side effects. The analgesic activity and opioid-sparing effects of pregabalin are negligibly affected by doses of either 300 or $600 \mathrm{mg}$, as shown previously by Apfelbaum et al. [6].

The immediate postoperative period is the most painful for patients undergoing microdiscectomy, a procedure that usually lasts 1-2 hours [25-28]; optimal CSF concentrations of drug may not be available during this most immediate and painful period for the patient [24]. In our study, we prescribed lower doses of both pregabalin and gabapentin than those used in previous studies, but the VAS values for pain 24 hours after surgery were comparable to those reported in the literature [29]. Similarly, both drugs provided comparable pain relief. 
We used the VAS to record postoperative pain control, as it has been shown to be a reliable, reproducible, and comparable objective scoring system by a number of investigators. It is considered the standard measurement tool for recording the extent of pain control. No patients required additional analgesia postoperatively, and no PCIA was used by any patient. This fact might contribute to the quality of the study, as the masking effects of morphine on analgesia and side effects were removed. The weakness of our study is that it lacked a placebo arm, which may have clarified any interactions between the drugs used in this study.

\section{Conclusions}

Preventive use of low-dose pregabalin is equivalent to that of gabapentin for the relief of postoperative pain in patients undergoing spine surgery. Therefore, we recommend the preferential use of pregabalin over gabapentin based on its superior pharmacokinetics and pharmacodynamics.

\section{Conflict of Interest}

No potential conflict of interest relevant to this article was reported.

\section{References}

1. Blumenthal S, Borgeat A, Nadig M, Min K. Postoperative analgesia after anterior correction of thoracic scoliosis: a prospective randomized study comparing continuous double epidural catheter technique with intravenous morphine. Spine (Phila Pa 1976) 2006; 31:1646-51.

2. Feldstein G, Ramanathan S. Obstetrical lumbar epidural anesthesia in patients with previous posterior spinal fusion for kyphoscoliosis. Anesth Analg 1985; 64:83-5.

3. Fisher CG, Belanger L, Gofton EG, et al. Prospective randomized clinical trial comparing patient-controlled intravenous analgesia with patient-controlled epidural analgesia after lumbar spinal fusion. Spine (Phila Pa 1976) 2003;28:739-43.

4. Kehlet H. Multimodal approach to control postoperative pathophysiology and rehabilitation. Br J Anaesth 1997;78:606-17.
5. Perkins FM, Kehlet H. Chronic pain as an outcome of surgery: a review of predictive factors. Anesthesiology 2000;93:1123-33.

6. Apfelbaum JL, Chen C, Mehta SS, Gan TJ. Postoperative pain experience: results from a national survey suggest postoperative pain continues to be undermanaged. Anesth Analg 2003;97:534-40.

7. Kumar RJ, Menon KV, Ranjith TC. Use of epidural analgesia for pain management after major spinal surgery. J Orthop Surg (Hong Kong) 2003;11:67-72.

8. Kehlet H, Dahl JB. The value of "multimodal" or "balanced analgesia" in postoperative pain treatment. Anesth Analg 1993;77:1048-56.

9. Acute pain management: operative or medical procedures and trauma, Part 2. Agency for Health Care Policy and Research. Clin Pharm 1992;11:391-414.

10. Dickenson AH. Neurophysiology of opioid poorly responsive pain. Cancer Surv 1994;21:5-16.

11. Ben-Menachem E. Pregabalin pharmacology and its relevance to clinical practice. Epilepsia 2004;45 Suppl 6:13-8.

12. Morello CM, Leckband SG, Stoner CP, Moorhouse DF, Sahagian GA. Randomized double-blind study comparing the efficacy of gabapentin with amitriptyline on diabetic peripheral neuropathy pain. Arch Intern Med 1999;159:1931-7.

13. Sabatowski R, Galvez R, Cherry DA, et al. Pregabalin reduces pain and improves sleep and mood disturbances in patients with post-herpetic neuralgia: results of a randomised, placebo-controlled clinical trial. Pain 2004;109:26-35.

14. Frampton JE, Foster RH. Pregabalin: in the treatment of postherpetic neuralgia. Drugs 2005;65:111-8.

15. Frampton JE, Scott LJ. Pregabalin: in the treatment of painful diabetic peripheral neuropathy. Drugs 2004; 64:2813-20.

16. Feltner DE, Crockatt JG, Dubovsky SJ, et al. A randomized, double-blind, placebo-controlled, fixeddose, multicenter study of pregabalin in patients with generalized anxiety disorder. J Clin Psychopharmacol 2003;23:240-9.

17. Pande AC, Feltner DE, Jefferson JW, et al. Efficacy of the novel anxiolytic pregabalin in social anxiety disorder: a placebo-controlled, multicenter study. J Clin Psychopharmacol 2004;24:141-9.

18. Pandey CK, Navkar DV, Giri PJ, et al. Evaluation of the optimal preemptive dose of gabapentin for 
postoperative pain relief after lumbar diskectomy: a randomized, double-blind, placebo-controlled study. J Neurosurg Anesthesiol 2005;17:65-8.

19. Burke SM, Shorten GD. Perioperative pregabalin improves pain and functional outcomes 3 months after lumbar discectomy. Anesth Analg 2010;110:1180-5.

20. UpToDate. Pregabalin: drug information [Internet]. Alphen aan den Rijn: Wolters Kluwer; 2016 [cited 2010 Jun 25]. Available from: http://www.uptodateonline.com/online/content/topic.do?topicKey=drug 1_z/107827\&selectedTitle=1\%7E38\&source=search_ result.

21. Pogatzki-Zahn EM, Zahn PK, Brennan TJ. Postoperative pain: clinical implications of basic research. Best Pract Res Clin Anaesthesiol 2007;21:3-13.

22. Savica R, Beghi E, Mazzaglia G, et al. Prescribing patterns of antiepileptic drugs in Italy: a nationwide population-based study in the years 2000-2005. Eur J Neurol 2007;14:1317-21.

23. Rose MA, Kam PC. Gabapentin: pharmacology and its use in pain management. Anaesthesia 2002;57: 451-62.

24. Buvanendran A, Kroin JS, Kari M, Tuman KJ. Can a single dose of $300 \mathrm{mg}$ of pregabalin reach acute an- tihyperalgesic levels in the central nervous system? Reg Anesth Pain Med 2010;35:535-8.

25. Asghar FA, Hilibrand AS. The impact of the Spine Patient Outcomes Research Trial (SPORT) results on orthopaedic practice. J Am Acad Orthop Surg 2012; 20:160-6.

26. Cummins J, Lurie JD, Tosteson TD, et al. Descriptive epidemiology and prior healthcare utilization of patients in the Spine Patient Outcomes Research Trial's (SPORT) three observational cohorts: disc herniation, spinal stenosis, and degenerative spondylolisthesis. Spine (Phila Pa 1976) 2006;31:806-14.

27. Jordan J, Konstantinou K, O’Dowd J. Herniated lumbar disc. BMJ Clin Evid 2009;2009:1118.

28. Morgan-Hough CV, Jones PW, Eisenstein SM. Primary and revision lumbar discectomy: a 16-year review from one centre. J Bone Joint Surg Br 2003;85:871-4.

29. Ozgencil E, Yalcin S, Tuna H, Yorukoglu D, Kecik Y. Perioperative administration of gabapentin 1,200 $\mathrm{mg}$ day- 1 and pregabalin $300 \mathrm{mg}$ day-1 for pain following lumbar laminectomy and discectomy: a randomised, double-blinded, placebo-controlled study. Singapore Med J 2011;52:883-9. 